\title{
Therapeutic effects of intravenous urapidil in elderly patients with hypertension and acute decompensated heart failure: A pilot clinical trial
}

\author{
WEI YANG ${ }^{1}$, YU-JIE ZHOU ${ }^{2}$, YAN FU ${ }^{3}$, JIAN QIN $^{4}$, SHU TAN $^{5}$, XIAO-MIN CHEN $^{6}$, JIN-CHENG GUO ${ }^{7}$, \\ DE-ZHAO WANG ${ }^{8}$, HONG ZHAN $^{9}$, WEI GUAN ${ }^{10}$, YA-WEI XU ${ }^{11}$, JING-YU HE ${ }^{4}$, JING $\mathrm{LI}^{4}$ and QI HUA ${ }^{4}$ \\ ${ }^{1}$ Department of Geriatrics, Xuan Wu Hospital, Capital Medical University, Beijing 100053; ${ }^{2}$ Department of Cardiology, \\ An Zhen Hospital Affiliated to Capital Medical University, Beijing 100029; ${ }^{3}$ Emergency Department, \\ Tong Ren Hospital Affiliated to Capital Medical University, Beijing 100005; ${ }^{4}$ Department of Cardiology, \\ Xuan Wu Hospital, Capital Medical University, Beijing 100053; ${ }^{5}$ Department of Cardiology, First Affiliated Hospital, \\ Chongqing Medical University, Chongqing 400016; ${ }^{6}$ Department of Cardiology, Ningbo First Hospital, Ningbo, \\ Zhejiang 315010; ${ }^{7}$ Department of Cardiology, Luhe Hospital, Affiliated to Capital Medial University, Beijing 101100; \\ ${ }^{8}$ Department of Cardiology, Mentougou District Hospital of Beijing City, Beijing 102300; ${ }^{9}$ Emergency Department, \\ The First Affiliated Hospital of Sun Yat-sen University, Guangzhou, Guangdong 510080; ${ }^{10}$ Department of Cardiology, \\ Heilongjiang Provincial Hospital, Harbin, Heilongjiang 150030; ${ }^{11}$ Department of Cardiology, \\ Shanghai Tenth People's Hospital, Tongji University School of Medicine, Shanghai 200072, P.R. China
}

Received February 22, 2015; Accepted April 5, 2016

DOI: $10.3892 /$ etm.2016.3302

\begin{abstract}
Urapidil has been proposed to be an effective vasodilator for the treatment of acute decompensated heart failure (ADHF); however, its effect on cardiac function, as compared with that of nitroglycerin, in elderly patients with hypertension and ADHF has yet to be determined. In the present study, a multicenter, open-label clinical trial was performed, in which 120 elderly patients with hypertension and ADHF were randomly assigned to the treatment (50-400 $\mu \mathrm{g} / \mathrm{min}$ intravenous urapidil) or control group (5-40 $\mu \mathrm{g} / \mathrm{min}$ intravenous nitroglycerin). The dosages of the medications were adjusted according to the blood pressure of the patients. The systolic and diastolic blood pressure, heart rate and serum level of $\mathrm{N}$-terminal pro B-type natriuretic peptide (NT-proBNP) were evaluated at hospital admission and at days 1, 2, 3 and 7 after treatment. In addition, the left ventricular function was assessed by measuring the left ventricular ejection fraction (LVEF) and left ventricular end-diastolic volume at hospital admission and at days 2 and 7 after treatment. The results indicated that intravenous administration of urapidil and nitroglycerin were effective in lowering the blood pressure and heart rate within 7 days, with no significant
\end{abstract}

Correspondence to: Dr Qi Hua, Department of Cardiology, Xuan Wu Hospital, Capital Medical University,45 Changchun Avenue, Xuanwu, Beijing 100053, P.R. China

E-mail: huaqi5371@medmail.com.cn

Key words: acute decompensated heart failure, urapidil, cardiac function, nitroglycerin, clinical trial differences observed between the two groups $(\mathrm{P}>0.05)$. By contrast, greater reduction in the serum NT-proBNP level $(2,410.4 \pm 546.1$ vs. $4,234.1 \pm 876.4 \mathrm{pg} / \mathrm{ml} ; \mathrm{P}<0.05)$ and greater improvement in the LVEF $(55.3 \pm 3.4$ vs. $45.2 \pm 2.4 \%$; $\mathrm{P}<0.05)$ were observed in the urapidil-treated group, as compared with the nitroglycerin-treated group. No adverse events were reported during the treatment period in the two groups. The clinical outcomes at 6 months following discharge were evaluated and were not found to be significantly different between the two groups. In conclusion, the present results of the present study suggested that urapidil was as effective as nitroglycerin in controlling blood pressure and heart rate and was more effective in improving cardiac systolic function in elderly patients with hypertension and ADHF.

\section{Introduction}

Despite significant improvements in the diagnosis and treatment of heart failure (HF), the mortality and morbidity rates for patients with HF remain high (1-3). For patients with hypertension, in particular elderly patients, the long-term overload of the heart caused by increased afterload, as well as reduced preservation of cardiac function, increases their susceptibility to cardiac insufficiency, which manifests clinically as acute decompensated HF (ADHF) (4). The hemodynamic characteristics of patients with ADHF have been shown to be different from those of patients with chronic $\mathrm{HF}$ (CHF) (5). In contrast to the reduced left ventricular systolic function observed in patients with CHF, patients with ADHF typically have elevated filling pressures, high systemic vascular resistance and hypertension, which may lead to pump failure and further reduction of the perfusion of blood to vital organs, resulting in vascular failure $(6,7)$. 
Therefore, vasodilators may be beneficial in these conditions due to their pharmacological actions of reducing cardiac afterload, as well as improving peripheral perfusion $(8,9)$.

Vasodilators have been considered to be an effective class of drugs in patients with hypertension and ADHF (10-12). The most commonly used vasodilator in patients with ADHF is nitroglycerin (11-13). Although nitroglycerin has been reported to be effective and applicable in the majority of cases, there have been reports regarding its unfavorable effect on the heart rate (HR) $(13,14)$. Furthermore, no evident benefits of nitroglycerin on the cardiac systolic and diastolic function have been reported (13). Therefore, there is an urgent requirement for the development of novel pharmacological agents for the treatment of hypertension and ADHF. Urapidil, which is a vasodilator that exerts peripheral $\alpha$-adrenergic receptor and central serotonin receptor 1A (5-HT1A) antagonizing effects, has been proposed to be potentially effective for patients with hypertension and $\operatorname{ADHF}(15,16)$. However, its effect on cardiac function, particularly in elderly patients with ADHF, has yet to be determined. Therefore, the aim of the current randomized controlled trial (RCT) was to compare the therapeutic effects of intravenous urapidil and nitroglycerin in elderly patients with hypertension and ADHF.

\section{Patients and methods}

Study design. The present study was designed as a multicenter clinical trial and was performed in 10 research centers across mainland China: Department of Cardiology and Emergency, Xuanwu Hospital of Capital Medical University (Beijing, China); Department of Geriatric Cardiology, Beijing Anzhen Hospital Affiliated to Capital Medical University (Beijing, China); Department of Internal Medicine, Beijing Tongren Hospital Affiliated to Capital Medical University (Beijing, China); Department of Cardiology, The First Affiliated Hospital of Chongqing Medical University (Chongqing, China); Department of Cardiology, Sir Run Run Shaw Hospital in Zhejiang Province (Hangzhou, China); Department of Cardiology, The Tenth People's Hospital Affiliated to Shanghai Tongji University (Shanghai, China); Department of Cardiology, Qilu Hospital of Shandong University (Jinan, China); Department of Cardiology, The First Hospital of Jilin University (Changchun, China); Department of Cardiology, The First Affiliated Hospital of Harbin Medical University (Harbin, China); and Department of Cardiology, Guangdong Provincial People's Hospital (Guangzhou, China). This open-label RCT aimed to evaluate the efficacy and safety of intravenous urapidil, as compared with that of the conventional vasodilator nitroglycerin, for the treatment of elderly hypertensive patients who also suffered from ADHF. Patients were enrolled in the present study between August 1st, 2011 and November 1st, 2013. The ethics committees of all the included research centers approved the protocols of the study prior to its initiation. All patients provided written informed consent prior to their enrollment. The protocol of the clinical trial was registered in the Chinese Clinical Trial Registry (no. ChiCTR-TRC-11001781) before the enrollment of the first patients. A subgroup study of present study, which included only AHF patients with DM, has been published previously (17).
Inclusion and exclusion criteria of the patients. The inclusion criteria for the present study were as follows: i) Age, $>65$ years; ii) a previous diagnosis of hypertension, defined as a systolic blood pressure (SBP) of $>140 \mathrm{mmHg}$ and/or a diastolic blood pressure (DBP) of $>90 \mathrm{mmHg}$, or regular use of antihypertensives (18); iii) a diagnosis of systolic ADHF; and iv) the patient had been scheduled for inpatient treatment at one of the included medical centers. The diagnosis of systolic ADHF was based on the decompensated clinical manifestations of the patients [New York Heart Association (NYHA) classification of II-IV (19)] and a reduced left ventricular ejection fraction (LVEF) of $<50 \%$, as assessed by transthoracic echocardiography (2).

Patients were excluded from the present study if they presented any of the following: i) Complications including cardiogenic shock, an SBP of $\leq 100 \mathrm{mmHg}$, cerebral ischemia or severe stenosis of the carotid arteries, or other clinical disorders that were contraindications for intravenous administration of vasodilators; ii) confirmed acute coronary syndrome; iii) comorbidities of severe structural heart diseases, including severe valvular stenosis, hypertrophic obstructive cardiomyopathy, restrictive cardiomyopathy or pericarditis; iv) a severe condition of other systems or organs, including severe chronic asthmatic bronchial and pulmonary diseases, severe liver dysfunction ( $>3$-fold maximum normal levels of alanine aminotransferase and aspartate aminotransferase) or kidney insufficiency ( $>2$-fold maximum normal level of creatinine); v) a known or suspected allergy to any of the tested medications and their ingredients; vi) unsuitable for participation as determined by researchers (for example, due to the presence of cancer cachexia or severe mental illness); vii) use of any of the tested medications within 60 days prior to enrollment; and viii) participation in another clinical research program.

Randomization, treatment assignment and medications. During the study process, all patients continued to receive conventional antihypertensives (such as calcium-channel blockers, angiotensin converting enzyme inhibitors, angiotensin receptor blockers and diuretics) and other cardiovascular medications (including digoxin, amiodarone and statins). However, $\beta$-blockers were not prescribed during the study period, since they may increase the risk of a deterioration of ADHF (19). The included patients were randomly assigned to either an urapidil-based treatment group or a nitroglycerin-based control group, according to a random number generated by a computer. The assignment of the patients was open to both the investigators and the participants.

For patients in the urapidil group, urapidil hydrochloride (100 mg diluted in $50 \mathrm{ml}$ normal saline; Ebrantil; Altana AG, Konstanz, Germany) was continuously administered intravenously as required and the dosages of the urapidil were adjusted according to the blood pressure of the patients. Typically, the starting dose of urapidil was set at a small dose in accordance with the clinical situation and then increased to the target dose (50-100 $\mu \mathrm{g} / \mathrm{min})$ within $6 \mathrm{~h}$, with a maximum dose of $400 \mu \mathrm{g} / \mathrm{min}$. Intravenous urapidil was discontinued if the SBP was $<90 \mathrm{mmHg}$. Furthermore, if the symptoms of ADHF were not improved during the initial $48 \mathrm{~h}$ of administration, the intravenous urapidil was discontinued and the subsequent treatment was based on the judgement of the 
Table I. Baseline characteristics of elderly hypertensive patients in both groups.

\begin{tabular}{|c|c|c|}
\hline Characteristic & Nitroglycerin group $(n=62)$ & Urapidil group $(n=58)$ \\
\hline Males & $36(58.1)$ & $34(58.6)$ \\
\hline Age (years) & $78.1 \pm 10.2$ & $78.0 \pm 10.0$ \\
\hline Duration of hypertension (years) & $17.1 \pm 10.6$ & $18.8 \pm 7.4$ \\
\hline $\operatorname{HGB}(\mathrm{g} / \mathrm{l})$ & $109.0 \pm 5.2$ & $107.2 \pm 4.6$ \\
\hline eGFR (ml/min) & $47.2 \pm 4.8$ & $45.0 \pm 6.2$ \\
\hline $\operatorname{LVEF}(\%)$ & $39.7 \pm 5.2$ & $41.9 \pm 4.2$ \\
\hline \multicolumn{3}{|l|}{ NYHA classification } \\
\hline Class II & $34(54.8)$ & $32(55.2)$ \\
\hline Class III & $18(29.0)$ & $16(27.6)$ \\
\hline Class IV & $10(16.2)$ & $10(17.2)$ \\
\hline \multicolumn{3}{|l|}{ Comorbidities } \\
\hline CHD & $18(29.0)$ & $17(29.3)$ \\
\hline $\mathrm{DM}$ & $27(43.5)$ & $26(44.8)$ \\
\hline AF & $10(16.1)$ & $11(18.9)$ \\
\hline \multicolumn{3}{|l|}{ Number of antihypertensives used } \\
\hline 1 & $15(24.2)$ & $17(29.3)$ \\
\hline 2 & $25(40.3)$ & $23(39.7)$ \\
\hline 3 & $22(35.5)$ & $18(31.0)$ \\
\hline \multicolumn{3}{|l|}{ Concurrent $\mathrm{CV}$ medications } \\
\hline $\mathrm{CCBs}$ & $48(77.4)$ & $46(79.3)$ \\
\hline ACEIs/ARBs & 37 (59.7) & $39(67.2)$ \\
\hline$\beta$-blockers & $18(29.0)$ & $16(27.8)$ \\
\hline Diuretics & $39(62.9)$ & $37(63.8)$ \\
\hline Digoxin & $17(27.4)$ & $14(24.1)$ \\
\hline Amiodarone & $12(19.4)$ & $13(22.4)$ \\
\hline Statins & $45(72.5)$ & $43(74.1)$ \\
\hline
\end{tabular}

Data are presented as the mean \pm standard deviation or $\mathrm{n}(\%)$. HGB, hemoglobin; eGFR, estimated glomerular filtration rate; LVEF, left ventricular ejection fraction; NYHA, New York Heart Association; CHD, coronary heart disease; DM, diabetes; AF, atrial fibrillation; CV, cardiovascular; CCB, calcium-channel blocker; ACEI, angiotensin converting enzyme inhibitor; ARB, angiotensin receptor blocker.

investigators. Similarly, for patients assigned to the nitroglycerin group, intravenous nitroglycerin $(10 \mathrm{mg}$ diluted in $50 \mathrm{ml}$ $5 \%$ glucose solution; Beijing Yimin Pharmaceutical Co., Ltd., Beijing, China) was continuously administered as required. The starting dose of nitroglycerin was set at a small dose in accordance with the clinical situation and then increased to the target dose $(5-10 \mu \mathrm{g} / \mathrm{min})$ within $6 \mathrm{~h}$, with a maximum dose of $40 \mu \mathrm{g} / \mathrm{min}$. The protocols for dosage adjustment and the criteria for medication discontinuation were the same as those for the intravenous urapidil group. Only patients who received the tested medications for $>24 \mathrm{~h}$ were included in subsequent analyses.

Study protocols. Each participant underwent repeated evaluations of hemodynamic parameters, including HR, SBP and DBP, at hospital admission (day 0) and at 1,2, 3 and 7 days after intravenous vasodilator administration was initiated, as previously described (17). In addition, serum levels of N-terminal B type natriuretic peptide (NT-proBNP) were evaluated at the same time points. The left ventricular function was evaluated by means of transthoracic echocardiography, while the parameters of LVEF and left ventricular end-diastolic volume (LVEDV) were also measured at hospital admission and at 2 and 7 days after intravenous vasodilator administration. Furthermore, the serum levels of indices that reflect the metabolism of glucose and lipids, including fasting plasma glucose (FPG), glycosylated hemoglobin (GHB), postprandial $2 \mathrm{~h}$ plasma glucose (P2hPG), triglycerin (TG), total cholesterol (TC), low-density lipoprotein cholesterol (LDL-C) and high-density lipoprotein cholesterol (HDL-C), were evaluated at hospital admission and on days 2 and 7 after intravenous vasodilator administration. Measurements of the above parameters were recorded as previously described (17). No other intravenous vasodilators were used during the study period.

Echocardiological evaluation of left ventricular function. Global left ventricular function was assessed by transthoracic echocardiography (Philips IE33 Ultrasound system; Philips Healthcare, Amsterdam, The Netherlands). The apical 2- and 
4-chamber views were selected for the measurement of LVEF, which was used as an index of global left ventricular function. To calculate the LVEF, the end-diastolic and end-systolic frames were selected, the end-diastolic and end-systolic endocardial borders were manually traced and the biplane LVEF was calculated. In addition, the LVEDV was measured for each patient, as previously described (17).

Follow-up and clinical outcomes. The patients were followed up for 6 months after discharge from the hospital. Clinical outcomes, including HF-associated rehospitalization, cardiovascular mortality, non-fatal myocardial infarction (MI) and malignant arrhythmia (including ventricular tachycardia and ventricular fibrillation) were evaluated for all patients.

Statistical analysis. Continuous data are presented as the mean \pm standard deviation and the categorical data are presented as the number and percentage. Each data set was assessed for normality. Differences in the continuous and categorical data between the two groups were analyzed using the Student's t-test or the $\chi^{2}$ test. Differences in the data from multiple time points between the two groups were analyzed using the repeated measures analysis of variance (ANOVA) and independent samples t-test. Statistical analyses were performed using SPSS version 16.0 software (SPSS, Inc., Chicago, IL, USA). P $<0.05$ was considered to indicate a statistically significant difference.

\section{Results}

Baseline characteristics of the included patients. A total of 120 elderly patients, including 70 males and 50 females, with hypertension and confirmed ADHF were included in the present study during the enrollment period from the 10 medical centers. The ages of the included patients ranged between 65 and 84 years. A total of 62 patients were randomly assigned to the nitroglycerin group, whereas the remaining 58 patients were assigned to the urapidil group. The baseline characteristics of the included patients are presented in Table I. There were no significant differences in the demographic factors, including age, gender, duration of hypertension, clinical manifestations of ADHF (as evaluated based on the LVEF and distribution of NYHA classification), comorbidities of coronary heart disease (CHD), diabetes mellitus and atrial fibrillation, as well as the use of cardiovascular medications, such as antihypertensives, digoxin, amiodarone and statins, between the two groups (all $\mathrm{P}>0.05$ ). All patients received predetermined protocols involving intravenous vasodilators and no adverse events were observed. The mean treatment duration for nitroglycerin was $87 \mathrm{~h}$ and the mean dosage was $88.7 \mathrm{mg}$ per patient. In comparison, the treatment duration for urapidil was $90 \mathrm{~h}$ and mean dosage was $405.2 \mathrm{mg}$ per patient.

Effect of urapidil versus nitroglycerin on $S B P, D B P$ and $H R$. A multivariate data analysis of the intragroup elements, using the repeated measures ANOVA, demonstrated that there were significant differences in the SBP $(\mathrm{F}=27.42)$, DBP $(\mathrm{F}=29.32)$ and $\mathrm{HR}(\mathrm{F}=31.24)$ among the various time points $(\mathrm{P}<0.05)$ in the two groups. This suggested that the hemodynamic parameters of SBP, DBP and HR were significantly lowered within
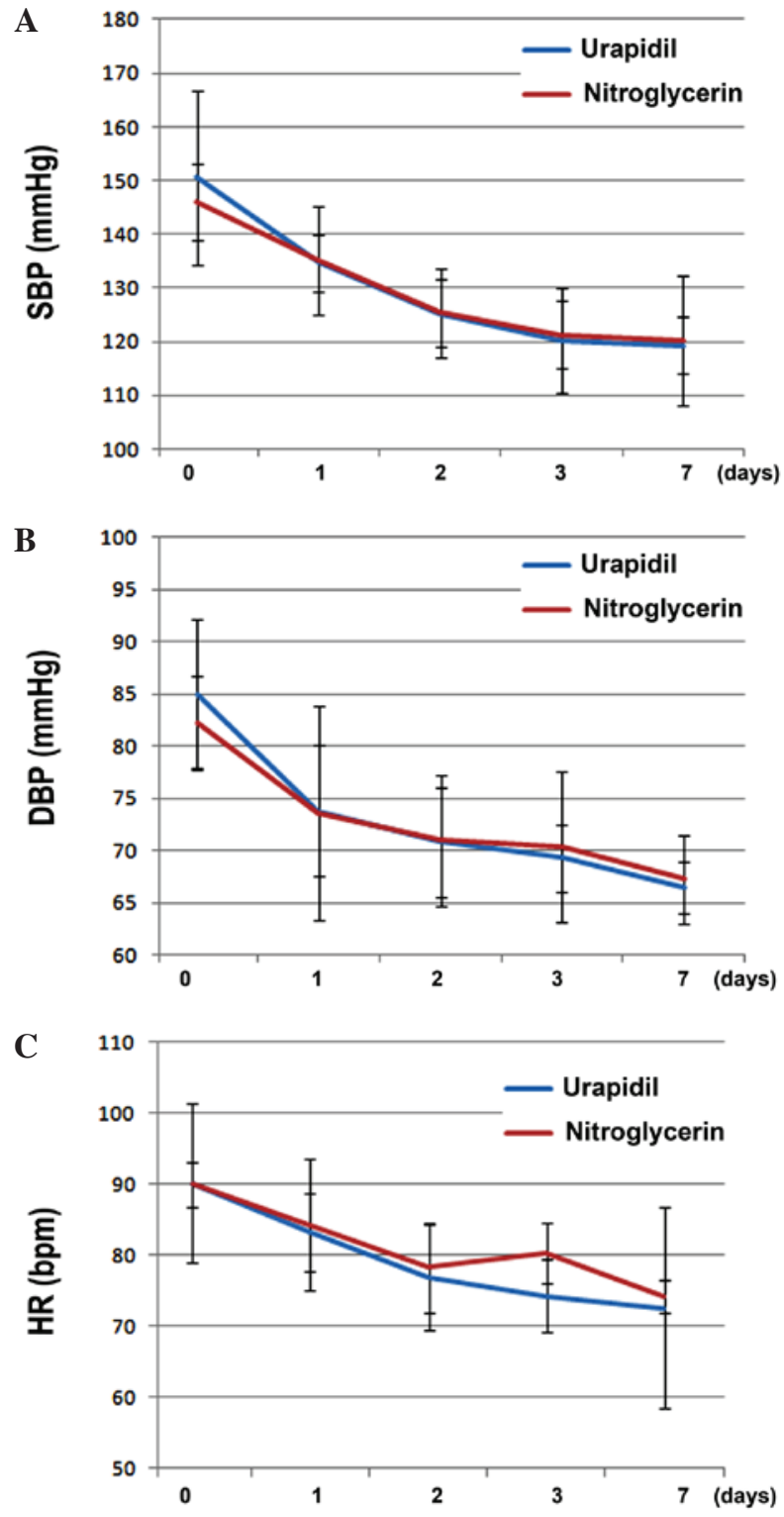

Figure 1. Effects of intravenous urapidil on (A) SBP, (B) DBP and (C) HR within 7 days of treatment, as compared with nitroglycerin, in elderly patients with hypertension and acute decompensated heart failure. SBP, systolic blood pressure; DBP, diastolic blood pressure; HR, heart rate.

7 days after intravenous administration of vasodilators, as compared with the values at admission (Fig. 1A-C). There was no association between time and group, which suggested that the time trends for the two medications were identical in their effects on the hemodynamic parameters of SBP, DBP and HR $(\mathrm{P}>0.05)$. Furthermore, intergroup ANOVA demonstrated that there were no significant differences regarding the effects of urapidil and nitroglycerin on the SBP, DBP and HR $(\mathrm{P}>0.05)$, thus suggesting that intravenous urapidil and nitroglycerin exerted similar blood pressure-lowering and HR-regulating effects within 7 days of treatment in elderly patients with hypertension and ADHF (Fig. 1A-C).

Effect of urapidil versus nitroglycerin on serum NT-proBNP levels. A multivariate analysis of intragroup elements, using the repeated measures ANOVA, demonstrated that the serum level of NT-proBNP (F=59.14) was significantly reduced after 


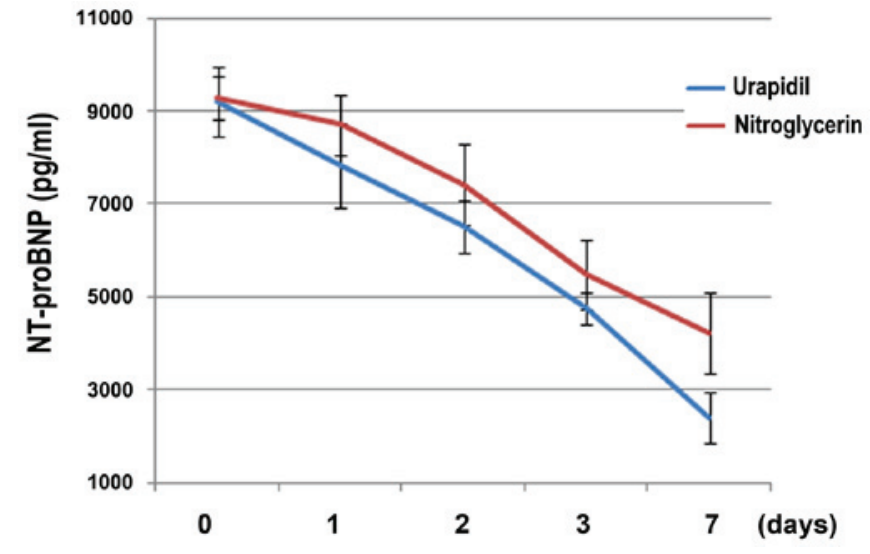

Figure 2. Effects of intravenous urapidil on the serum level of NT-proBNP within 7 days of treatment, as compared with nitroglycerin, in elderly patients with hypertension and acute decompensated heart failure. NT-proBNP, $\mathrm{N}$-terminal pro B type natriuretic peptide.

7 days of treatment with intravenous vasodilators in the two groups, as compared with the level at admission $(\mathrm{P}<0.05$; Fig. 2). However, there was no significant association between time and group, which indicated that the time trends for the two medications were identical in their effects on the serum levels of NT-proBNP $(\mathrm{P}>0.05)$. Intergroup ANOVA demonstrated that urapidil $(\mathrm{F}=57.24)$ was able to reduce the serum levels of NT-proBNP to a greater extent when compared with nitroglycerin $(\mathrm{P}<0.05)$, thus suggesting that intravenous urapidil may be more effective in improving cardiac function in elderly patients with hypertension and ADHF (Fig. 2).

Effect of urapidil versus nitroglycerin on LVEF and LVEDV. The effect of urapidil, as compared with nitroglycerin, on the index of left ventricular function, LVEF, are presented in Fig. 3A. Although no significant differences were observed in the LVEF between the two groups at admission and 2 days after intravenous vasodilator treatment, the LVEF for patients allocated to the urapidil group was significantly improved at 7 days after treatment, as compared with the nitroglycerin group (55.3 \pm 3.4 vs. $45.2 \pm 2.4 \%$, respectively; $\mathrm{t}=-3.104 ; \mathrm{P}<0.05$; Fig. 3A). These results indicate that urapidil exerts a more beneficial effect on left ventricular function, as compared with nitroglycerin. Conversely, no significant differences were detected in the LVEDV between the two groups at admission or on days 2 and 7 after treatment with intravenous vasodilators (all $\mathrm{P}>0.05$; Fig. 3B).

Effect of urapidil on the serum indices of glucose and lipid metabolism. The independent samples t-test was used to compare the differences in glucose metabolism indices, including FPG, P2hPG and GHB, in the patients with hypertension and ADHF at admission and on days 2 and 7 after intravenous vasodilator treatment. As compared with the nitroglycerin group, patients in the urapidil group showed no significant differences in any of the glycemic indices on days 2 and 7 after treatment (all P>0.05; Table II); however, a greater reducing trend in the urapidil group, as compared with the nitroglycerin group, could be predicted from the changes in the glycemic data. Similarly, the serum levels of lipids, including TG, TC, LDL-C and HDL-C, were not significantly
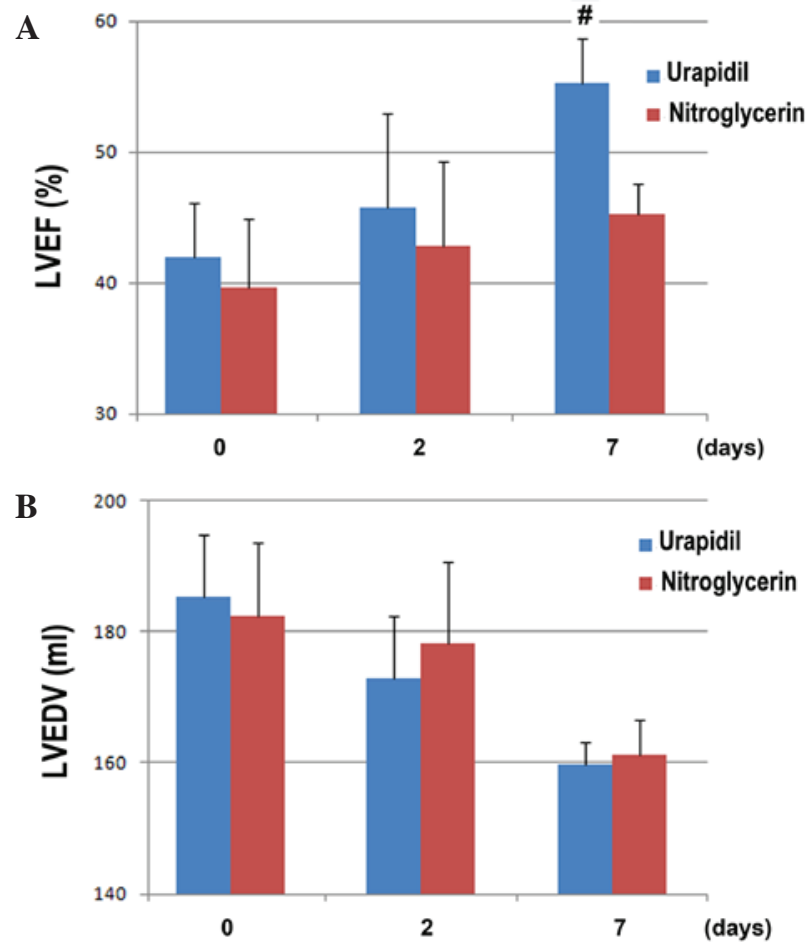

Figure 3. Effects of intravenous urapidil on the (A) LVEF and (B) LVEDV within 7 days of treatment, as compared with nitroglycerin, in elderly patients with hypertension and acute decompensated heart failure. LVEF, left ventricular ejection fraction; LVEDV, left ventricular end-diastolic volume. ${ }^{\text {"}} \mathrm{P}<0.05$ vs. nitroglycerin group.

different between the two groups at admission and on days 2 and 7 after treatment (all $\mathrm{P}>0.05$; Table II).

Follow-up data within 6 months following discharge. The follow-up data for patients in the urapidil and nitroglycerin groups showed no significant differences regarding the incidences of rehospitalization for HF, cardiovascular-associated mortality, non-fatal myocardial infarction or malignant arrhythmia (Table III).

\section{Discussion}

The present multicenter, controlled clinical trial compared the therapeutic effects of intravenous administration of urapidil and nitroglycerin in elderly patients with ADHF and hypertension. The results indicated that intravenous urapidil exerted similar blood pressure-lowering effects as nitroglycerin in patients with ADHF. Furthermore, the two treatments were not associated with an evident increase in HR and there were no reports of acute adverse events. Notably, short-term administration of urapidil was associated with a greater improvement in cardiac systolic function compared with nitroglycerin, as demonstrated by the greater extent by which urapidil increased the LVEF and reduced the serum level of NT-proBNP level on day 7 of treatment in elderly patients with ADHF. However, the follow-up data suggested that the clinical outcomes of the patients in the two groups were not significantly different. These results suggested that, as compared with intravenous nitroglycerin, intravenous urapidil may be more effective for improving cardiac systolic function in elderly patients with hypertension 
Table II. Effects of urapidil on indices of glucose and lipid metabolism, compared with the effect of nitroglycerin.

\begin{tabular}{|c|c|c|c|c|c|c|}
\hline \multirow[b]{2}{*}{ Index } & \multicolumn{3}{|c|}{ Nitroglycerin group $(n=62)$} & \multicolumn{3}{|c|}{ Urapidil group $(\mathrm{n}=58)$} \\
\hline & Baseline & 2 days & 7 days & Baseline & 2 days & 7 days \\
\hline FPG (mmol/l) & $7.86 \pm 4.68$ & $7.28 \pm 2.34$ & $6.99 \pm 2.34$ & $7.99 \pm 1.24$ & $7.55 \pm 2.12$ & $6.12 \pm 2.56$ \\
\hline GHB (mmol/l) & $7.14 \pm 2.09$ & $7.05 \pm 3.34$ & $6.93 \pm 1.47$ & $7.16 \pm 1.24$ & $6.74 \pm 3.52$ & $6.53 \pm 1.24$ \\
\hline $\mathrm{P} 2 \mathrm{hPG}(\mathrm{mmol} / \mathrm{l})$ & $11.29 \pm 5.44$ & $10.61 \pm 5.62$ & $10.04 \pm 1.25$ & $11.52 \pm 4.08$ & $10.66 \pm 1.52$ & $9.25 \pm 3.51$ \\
\hline $\mathrm{TG}(\mathrm{mmol} / \mathrm{l})$ & $1.38 \pm 1.30$ & $1.43 \pm 0.27$ & $1.40 \pm 0.68$ & $1.44 \pm 0.26$ & $1.38 \pm 0.76$ & $1.37 \pm 0.58$ \\
\hline $\mathrm{TC}(\mathrm{mmol} / \mathrm{l})$ & $3.93 \pm 1.04$ & $3.88 \pm 0.91$ & $4.06 \pm 1.01$ & $4.11 \pm 1.12$ & $4.07 \pm 1.36$ & $4.04 \pm 1.31$ \\
\hline LDL-C (mmol/l) & $2.37 \pm 0.34$ & $2.32 \pm 0.75$ & $2.29 \pm 0.81$ & $2.91 \pm 1.16$ & $2.78 \pm 1.26$ & $2.78 \pm 1.23$ \\
\hline HDL-C (mmol/l) & $1.13 \pm 0.23$ & $1.12 \pm 0.34$ & $1.16 \pm 0.21$ & $1.13 \pm 0.46$ & $1.23 \pm 0.24$ & $1.26 \pm 0.36$ \\
\hline
\end{tabular}

Data are presented as the mean \pm standard deviation. FPG, fasting plasma glucose; GHB, glycosylated hemoglobin; P2hPG, postprandial $2 \mathrm{~h}$ plasma glucose; TG, triglycerin; TC; total cholesterol; LDL-C, low-density lipoprotein cholesterol; HDL-C, high-density lipoprotein cholesterol.

Table III. Incidence of adverse cardiovascular events during the 6-month follow-up.

\begin{tabular}{lcc}
\hline Index & Nitroglycerin group $(\mathrm{n}=62)$ & Urapidil group $(\mathrm{n}=58)$ \\
\hline Rehospitalization for heart failure & $12(19.4)$ & $7(12.1)$ \\
Cardiovascular-related death & $2(3.2)$ & $1(1.7)$ \\
Non-fatal myocardial infarction & $4(6.5)$ & $1(1.7)$ \\
Malignant arrhythmia & $3(4.8)$ & $2(3.4)$ \\
\hline
\end{tabular}

Data are presented as $\mathrm{n}(\%)$.

and ADHF. Further large-scale RCTs are required in order to determine whether the acute beneficial effects of urapidil on cardiac function lead to improved clinical outcomes for elderly patients with ADHF and hypertension in the long-term.

Previous epidemiological studies and registry trials reported that ADHF, which is characterized by acute insufficiency of cardiac systolic function and failure of peripheral perfusion of organs, has emerged as one of the most important causes of mortality and morbidity in the elderly population $(2,3,19)$. In China, the outcome of acute HF is typically poor, with a hospital mortality rate of $3 \%$, a 6-month readmission rate of $\leq 50 \%$ and a 5 -year mortality rate as high as $60 \%$ (2). Therefore, there is an urgent requirement for the development of novel treatment strategies for patients with ADHF. The majority of ADHF cases are complicated by elevated blood pressure, which may cause overloading of the heart and increased peripheral vascular resistance, thereby exacerbating the hemodynamic conditions $(6,20)$. Vasodilators have been recommended as the first-line medication for patients with ADHF, which may be due to their ability to stabilize the hemodynamic status $(4,9)$. The present study demonstrated that intravenous administration of urapidil and nitroglycerin conferred similar blood pressure-lowering effects in elderly patients with ADHF and hypertension, without having adverse effects on HR. These results are consistent with those of previous studies, which similarly reported the beneficial effects of urapidil and nitroglycerin on the hemodynamic status in patients with ADHF $(16,21,22)$. In a previous study investigating patients with CHF, $25 \mathrm{mg}$ intravenous urapidil administered twice within 15 min was associated with a significant decrease of $16 \%$ in the SBP, $13 \%$ in the mean arterial pressure, $38 \%$ in the left ventricular end-diastolic pressure, $31 \%$ in the mean pulmonary artery pressure and $40 \%$ in the wedge pressure (16). The mechanisms underlying the beneficial effects of urapidil on hemodynamic conditions have been considered based on its elucidated pharmacological characteristics, including antagonizing effects on the peripheral $\alpha$-adrenergic receptor and 5-HT1A, which enable it to be effective in the regulation of heart overloading (15).

As well as stabilizing the hemodynamic status of patients with ADHF and hypertension, another important therapeutic strategy for these patients is to improve the cardiac systolic function $(23,24)$. An early animal study (25) and observational studies $(16,22,26,27)$ reported potential beneficial effects of urapidil on cardiac output; however, its effect on the left ventricular systolic function in patients with ADHF has not been systematically evaluated in controlled clinical trials. To the best of our knowledge, the present study was the first to evaluate the effects of acute administration of urapidil on cardiac function, as compared with nitroglycerin administration, in ADHF patients in a randomized clinical trial. The results demonstrated that, on day 7 after treatment, patients who received intravenous urapidil exhibited a greater improvement in left systolic function, as compared with those who received nitroglycerin, which was shown by the greater improvement of the LVEF and a greater reduction in the serum level of NT-proBNP. LVEF and serum NT-proBNP have been confirmed to be reflective of cardiac systolic function in patients 
with $\operatorname{ADHF}(28,29)$. Furthermore, these indices were shown to have a prognostic value in these patients (30). The results of the present study were consistent with those of a previous study that showed that long-term urapidil administration was able to improve the LVEF in the 3 months following coronary revascularization in patients with CHD who had undergone coronary stenting (31). The exact mechanisms underlying the beneficial effects of urapidil on cardiac function are currently unknown. However, a previous study in patients with acute coronary syndrome suggested that urapidil treatment may improve the left ventricular function by increasing the coronary flow and myocardial perfusion, thereby improving the energy metabolism of the myocardium (32).

The present study observed that there were no significant differences in the effects of urapidil and nitroglycerin on the indices of glucose and lipid metabolism on day 7 after treatment between the two groups. Previous studies have suggested potential benefits of urapidil against insulin resistance in patients with hypertension (33); however, its metabolic benefits in patients with ADHF need to be observed in further studies. Furthermore, the long-term influence of intravenous urapidil on clinical outcomes requires clarification in large-scale RCTs in the future.

However, the current study had several limitations that must be considered when interpreting the results. Firstly, the study was designed as an open-label trial, which may lead to bias and the results of the study may, therefore, have been affected by subjective factors introduced by the patients and researchers. Furthermore, the study was designed as a pilot study and only a limited number of patients was included. Therefore, the possibility of the lack of statistical power in the present study can not be excluded and certain negative results should be interpreted with caution. Finally, differences in the effects of urapidil on patients with ischemia and without ischemia were not evaluate due to the limited number of patients included. Therefore, further high-quality, large-scale RCTs are required.

In conclusion, the present study demonstrated that intravenous urapidil was as effective as nitroglycerin in lowering blood pressure and was more effective in improving cardiac systolic function in elderly patients with hypertension and ADHF, suggesting that urapidil may be more a more effective therapeutic strategy than nitroglycerin in hypertensive patients with ADHF. Further large-scale RCTs are required to confirm the results and to observe the long-term effects of urapidil on clinical outcomes in these patients.

\section{Acknowledgements}

The present study was supported by a grant from the Capital Medical Development Foundation (863 project no. 2012BAI37B03).

\section{References}

1. Jiang $\mathrm{H}$ and $\mathrm{Ge} \mathrm{J}$ : Epidemiology and clinical management of cardiomyopathies and heart failure in China. Heart 95: 1727-1731, 2009.

2. Chinese Society of Cardiology of Chinese Medical Association; Editorial Board of Chinese Journal of Cardiology: Chinese guidelines for the diagnosis and treatment of heart failure 2014 Zhonghua Xin Xue Guan Bing Za Zhi 42: 98-122, 2014 (In Chinese).
3. Mozaffarian D, Benjamin EJ, Go AS, Arnett DK, Blaha MJ, Cushman M, de Ferranti S, Després JP, Fullerton HJ, Howard VJ, et al; American Heart Association Statistics Committee and Stroke Statistics Subcommittee: Heart disease and stroke statistics - 2015 update: A report from the American Heart Association. Circulation 131: e29-322, 2015.

4. Cicci JD, Reed BN, McNeely EB, Oni-Orisan A, Patterson JH and Rodgers JE: Acute decompensated heart failure: Evolving literature and implications for future practice. Pharmacotherapy 34: 373-388, 2014.

5. Felker GM, Adams KF Jr, Konstam MA, O'Connor CM and Gheorghiade M: The problem of decompensated heart failure: Nomenclature, classification and risk stratification. Am Heart J 145 (Suppl 2): S18-S25, 2003.

6. Adams KF Jr, Fonarow GC, Emerman CL, LeJemtel TH, Costanzo MR, Abraham WT, Berkowitz RL, Galvao M and Horton DP; ADHERE Scientific Advisory Committee and Investigators: Characteristics and outcomes of patients hospitalized for heart failure in the United States: rationale, design and preliminary observations from the first 100,000 cases in the Acute Decompensated Heart Failure National Registry (ADHERE). Am Heart J 149: 209-216, 2005.

7. Zannad F, Mebazaa A, Juillière Y, Cohen-Solal A, Guize L, Alla F, Rougé P, Blin P, Barlet MH, Paolozzi L, et al: Clinical profile, contemporary management and one-year mortality in patients with severe acute heart failure syndromes: The EFICA study. Eur J Heart Fail 8: 697-705, 2006.

8. Piper S and McDonagh T: The role of intravenous vasodilators in acute heart failure management. Eur J Heart Fail 16: 827-834, 2014.

9. Levy PD, Laribi S and Mebazaa A: Vasodilators in acute heart failure: Review of the latest studies. Curr Emerg Hosp Med Rep 2: 126-132, 2014.

10. Elkayam U, Janmohamed M, Habib M and Hatamizadeh P: Vasodilators in the management of acute heart failure. Crit Care Med 36 (Suppl 1): S95-S105, 2008.

11. Hollenberg SM: Vasodilators in acute heart failure. Heart Fail Rev 12: 143-147, 2007.

12. Metra M, Teerlink JR, Voors AA, Felker GM, Milo-Cotter O, Weatherley B, Dittrich $\mathrm{H}$ and Cotter G: Vasodilators in the treatment of acute heart failure: What we know, what we don't. Heart Fail Rev 14: 299-307, 2009.

13. Elkayam U, Bitar F, Akhter MW, Khan S, Patrus S and Derakhshani M: Intravenous nitroglycerin in the treatment of decompensated heart failure: Potential benefits and limitations. J Cardiovasc Pharmacol Ther 9: 227-241, 2004.

14. den Uil CA and Brugts JJ: Impact of intravenous nitroglycerin in the management of acute decompensated heart failure. Curr Heart Fail Rep 12: 87-93, 2015.

15. Buch J: Urapidil, a dual-acting antihypertensive agent: Current usage considerations. Adv Ther 27: 426-443, 2010.

16. Tebbe U, Wurst W and Neuhaus KL: Acute haemodynamic effects of urapidil in patients with chronic left ventricular failure. Eur J Clin Pharmacol 35: 305-308, 1988.

17. Yang W, Zhou YJ, Fu Y, Qin J, Qin S, Chen XM, Guo JC and Hua Q: A multicenter, randomized, trial comparing urapidil and nitroglycerin in multifactor heart failure in the elderly. Am J Med Sci 350: 109-115, 2015

18. Liu LS; Writing Group of 2010 Chinese Guidelines for the Management of Hypertension: 2010 Chinese guidelines for the management of hypertension. Zhonghua Xin Xue Guan Bing Za Zhi 39: 579-615, 2011 (In Chinese).

19. Yancy CW, Jessup M, Bozkurt B, Butler J, Casey DE, Drazner MH, Fonarow GC, Geraci SA, Horwich T, Januzzi JL and Johnson MR: 2013 ACCF/AHA guideline for the management of heart failure: A report of the American College of Cardiology Foundation/American Heart Association Task Force on practice guidelines. J Am Coll Cardiol 62: e147-239, 2013.

20. Cherney D and Straus S: Management of patients with hypertensive urgencies and emergencies: a systematic review of the literature. J Gen Intern Med 17: 937-945, 2002.

21. Zink M, Gombotz H, Wasler A, Grasser B, Rehak P and Metzler H: Urapidil reduces elevated pulmonary vascular resistance in patients before heart transplantation. J Heart Lung Transplant 21: 347-353, 2002.

22. Drobinski G, Montalescot G, Fossier JM and Grosgogeat Y: Effects of urapidil by intravenous injection on pulmonary circulation and cardiac function in left ventricular failure. Ann Cardiol Angeiol (Paris) 42: 167-172, 1993. 
23. Carlson MD and Eckman PM: Review of vasodilators in acute decompensated heart failure: The old and the new. J Card Fail 19: 478-493, 2013.

24. Fermann GJ and Collins SP: Initial management of patients with acute heart failure. Heart Fail Clin 9: 291-301, 2013.

25. Carlyle PF and Cohn JN: Systemic and regional hemodynamic effects of alpha-adrenoceptor blockade in chronic left ventricular dysfunction in the conscious dog. Am Heart J 120: 619-624, 1990.

26. Wang RY, Chow JS, Chan KH, Pan HY and Wong RP: Acute haemodynamic and myocardial metabolic effects of intravenous urapidil in severe heart failure. Eur Heart J 5: 745-751, 1984.

27. M'Buyamba-Kabangu JR, Bielen E, Staessen J, Fagard R, Lijnen P, Van Hoof R and Amery A: Hemodynamic effects of urapidil in men. Presse Med 19: 1407-1411, 1990.

28. Toma M, Ezekowitz JA, Bakal JA, O'Connor CM Hernandez AF, Sardar MR, Zolty R, Massie BM, Swedberg K and Armstrong PW: The relationship between left ventricular ejection fraction and mortality in patients with acute heart failure: Insights from the ASCEND-HF Trial. Eur J Heart Fail 16: 334-341, 2014.
29. Bhardwaj A and Januzzi JL Jr: Natriuretic peptide-guided management of acutely destabilized heart failure: Rationale and treatment algorithm. Crit Pathw Cardiol 8: 146-150, 2009.

30. Parissis JT, Nikolaou M, Mebazaa A, Ikonomidis I, Delgado J, Vilas-Boas F, Paraskevaidis I, Mc Lean A, Kremastinos D and Follath F: Acute pulmonary oedema: Clinical characteristics, prognostic factors and in-hospital management. Eur J Heart Fail 12: 1193-1202, 2010.

31. Kozàkovà M, Marco J,Heusch G, Bernies M, Bossi IM,Palombo C, Anguissola GB, Donatelli F, Laurent JP and Gregorini L: The alpha1-adrenergic blocker urapidil improves contractile function in patients 3 months after coronary stenting: A randomized, double-blinded study. Am Heart J 147: E6, 2004.

32. Yao DK, Jia SQ, Wang L, Li HW, Zhang YC, Wang YL and Wang LX: Therapeutic effect of urapidil on myocardial perfusion in patients with ST-elevation acute coronary syndrome. Eur J Intern Med 20: 152-157, 2009.

33. Oren S, Turkot S, Paran E, Flandra O, Slezak L and Hof B: Efficacy and tolerability of slow release urapidil (ebrantil) in hypertensive patients with non-insulin dependent diabetes mellitus (NIDDM). J Hum Hypertens 10: 123-127, 1996. 